\title{
GGADN: Guided Generative Adversarial Dehazing Network
}

\author{
Zhang Jian ( 41789332@qq.com ) \\ Wuhan Sports University \\ Wanjuan Song \\ Hubei University of Education
}

\section{Research Article}

Keywords: Dehazing, GAN, Guidance, Loss function

Posted Date: April 7th, 2021

DOl: https://doi.org/10.21203/rs.3.rs-386958/v1

License: (c) (i) This work is licensed under a Creative Commons Attribution 4.0 International License. Read Full License

Version of Record: A version of this preprint was published at Soft Computing on August 3rd, 2021. See the published version at https://doi.org/10.1007/s00500-021-06049-w. 


\title{
GGADN: Guided Generative Adversarial Dehazing Network
}

\author{
Jian Zhang • Wanjuan Song
}

Received: date / Accepted: date

\begin{abstract}
Image dehazing has always been a challenging topic in image processing. The development of deep learning methods, especially the Generative Adversarial Networks(GAN), provides a new way for image dehazing. In recent years, many deep learning methods based on GAN have been applied to image dehazing. However, GAN has two problems in image dehazing. Firstly, For haze image, haze not only reduces the quality of the image, but also blurs the details of the image. For Gan network, it is difficult for the generator to restore the details of the whole image while removing the haze. Secondly, GAN model is defined as a minimax problem, which weakens the loss function. It is difficult to distinguish whether GAN is making progress in the training process. Therefore, we propose a Guided Generative Adversarial Dehazing Network(GGADN). Different from other generation adversarial networks, GGADN adds a guided module on the generator. The guided module verifies the network of each layer of the generator. At the same time, the details of the map generated by each layer are strengthened. Network training is based on the pre-trained VGG feature model and L1-regularized gradient prior which is developed by new loss function parameters. From the dehazing results of synthetic images and real images, proposed method is better than the state-of-the-art dehazing methods.
\end{abstract}

Keywords Dehazing · GAN · Guidance · Loss function

J. Zhang

College of Sport Engineering and Information Technology, Wuhan Sports University, Wuhan, China

W. Song

College of Computer, Hubei University of Education, Wuhan, Hubei, China

E-mail: key_swj@whu.edu.cn 


\section{Introduction}

In computer vision, weather is an important factor affecting the quality of image[1-6]. Especially for haze, floating particles in haze lead to the fading and blurring of pictures, and the reduction of contrast and softness. They absorb and scatter light, resulting in serious color attenuation, poor clarity and contrast, and poor visual effect, which has a serious impact on subsequent computer vision tasks[7-16]. Therefore, it is necessary to remove haze effectively.

In recent years, the research of image dehazing algorithm has made great progress. At present, image dehazing research is mainly divided into two types, feature-based method and learning-based method. The difficulty of featurebased method lies in the feature extraction and a priori choice[17-20]. The common dehazing features and priors are as follows:

- Contrast: Tan found that the contrast of haze free image was high. Thus, image dehazing is performed by maximizing the local contrast of the image.

- Dark channel priori: He found that the value of dark channel in haze free image is close to zero, and then it can be used to estimate the transmission image.

- The prior of color attenuation: Zhu found the relationship between haze concentration and brightness and saturation through statistics. And a linear model of scene depth is created to solve the scene. Then, the haze-free image is calculated.

Learning based dehazing algorithms can be divided into two kinds, stepby-step learning algorithm and end-to-end learning algorithm. Step by step learning algorithm is similar to the traditional method, focusing on the prediction of intermediate variables. For example, Cai [21] designed a dehazenet by analyzing artificial prior features to complete the prediction of transmission image. Similarly, Ren [22] proposed a multi-scale convolutional neural network mscnn, which can accurately predict the transmission image through two different scale network models. End-to-end learning algorithm can realize image dehazing simply and efficiently through the design of full convolutional neural network. For example, considering that the above algorithm ignores the reasonable prediction of atmospheric light value, $\mathrm{Li}$ [23] integrated multiple intermediate variables in the atmospheric scattering model into one using linear variation, and proposed AOD net to directly predict haze-free images.

In this paper, we presents a Guided Generative Adversarial Dehazing Network(GGADN). Different from other generation adversarial networks, GGADN adds a guided module on the generator. The guided module verifies the network of each layer of the generator. At the same time, the details of the map generated by each layer are strengthened. GGADN is trained and corrected in a synthetic fuzzy image dataset containing indoor and outdoor images. Network training is based on the pre-trained Visual Geometry Group (VGG) feature model and L1-regularized gradient prior which is developed by new loss function parameters. From the dehazing results of synthetic images and real 
images, proposed method is better than the state-of-the-art dehazing methods. And the image after dehazing is clearer in detail.

\section{Related Works}

\subsection{Atmospheric Scattering Model}

The purpose of image defogging is to restore a clear image from the blurred image corroded by haze or smoke. In the field of computer vision, in order to overcome the image distortion caused by haze, McCartney [24] proposed an atmospheric scattering model which can be used to describe the formation process of haze image [25-27]. The equation is as follow.

$$
I(x)=J(x) t(x)+A(1-t(x))
$$

Where, $I(x)$ is a haze image and $J(x)$ is a haze-free image. $A(x)$ is the value of atmospheric light, representing the intensity of atmospheric light. $T(x)$ is the transmittance, which indicates the part of light that is not scattered when it reaches the imaging device through the atmospheric medium, and $x$ represents the pixel position. In the formula, the first term on the right is the direct attenuation term, which represents the reflected light of the object after atmospheric attenuation, and the second term is the enhanced atmospheric light obtained by atmospheric scattering.

When the composition of the atmosphere is uniform, that is, $A(x)$ is constant, the transmittance can be expressed as:

$$
t(x)=e^{-\beta d(x)}
$$

Where $\beta$ is the attenuation coefficient of the atmosphere and $D(x)$ is the depth of the scene. From the formula, it is not difficult to find that the scene depth and atmospheric light value have a great influence on the image dehazing effect. In single image dehazing, only the haze image is known, the atmospheric light value and scene depth are unknown, and the assumption of uniform atmospheric composition is not necessarily true. Therefore, how to remove haze effectively is a challenging problem.

\subsection{Generative Adversarial Networks}

Gan(Generative adversarial networks) is a deep learning model designed by Goodfellow [28]. Gan is a deep learning model, which is one of the most promising unsupervised learning methods on complex distribution in recent years [29-35]. The model produces good output through the game learning of two modules in the framework: Generative model and Discriminative model. In the original Gan theory, we don't need that G and D are neural networks, we just need to fit the corresponding generating and discriminating functions. But in 
practice, deep neural networks are generally used as G and D. An excellent Gan application needs a good training method, otherwise the output may not be ideal due to the freedom of the neural network model.

However, the network structure of Gan is unstable in the training process, and some artifacts such as noise and color shift are often produced in the synthetic image. Sohn [36] introduced conditional information into Gan. The increase of conditional information variables ensures the stability of learning process to a certain extent and improves the representation ability of generator, but the running time is too long. Deniz [37] propose an end-to-end network called Cycle-Dehaze for single image dehazing. In order to improve the quality of texture information recovery and produce a better visual haze free image, the CycleGan agent is enhanced by combining cycle consistency and perceptual loss. Du [38] also uses the end-to-end learning method to learn the mapping from hazy image to haze free image directly. This dehazing network generates the confrontation training network through the Gan model. An adaptive loss function is used in discriminator. A post-processing method for halo artifacts removal using guided filtering is proposed.

Although the existing Gan network has achieved some results in haze removal, there are also some problems. Firstly, Gan model is defined as a minimax problem, which has no loss function. It is difficult to distinguish whether Gan is making progress in the training process. The learning process of Gan may have collapse problem, and the generator begins to degenerate. It always generates the same sample points and cannot continue learning. When the generating model collapses, the discrimination model will also point to the similar direction for the similar sample points, so the training cannot continue. Secondly, For haze image, haze not only reduces the quality of the image, but also blurs the details of the image. For Gan network, it is difficult for the generator to restore the details of the whole image while removing the haze. Especially for the image with complex structure, the effect of dehazing is not ideal.

In view of the above problems, this paper improves the dehazing Gan and designs a Guided Generative Adversarial Dehazing Network(GGADN).

\section{The proposed method}

This paper presents a Guided Generative Adversarial Dehazing Network(GGADN).

In GGADN the generator and discriminator architecture are modified. The synthetic data set is trained by end-to-end training neural network. The loss function is modified by using the pre-trained VGG feature and L1-regularization gradient. Sigmoid function is introduced to the last layer of discriminator for feature mapping. In order to carry out probability analysis, the discriminant results can be normalized to $[0,1]$. 


\subsection{Guided Generative Adversarial Dehazing Network(GGADN)}

In the proposed algorithm, an end-to-end dehazing network is used to train the network to avoid image distortion or artifact which caused by the estimation of transmittance and atmospheric light value. In order to generate better dehazing image from generator, generator and discriminator architecture are designed in the GGan network so that it can capture more useful information. At the same time, for generating realistic and clear images and remove artifacts, the dehazing network is trained and corrected in a synthetic fuzzy image dataset containing indoor and outdoor images. Network training is based on the pre-trained VGG feature model and L1-regularized gradient prior which is developed by new loss function parameters.

The VGG features include:

- Small convolution core, replacing convolution core with $3 \times 3$.

- Small pool core, replacing $3 \times 3$ pool core with $2 \times 2$ pool core.

- Deeper layer feature map and wider feature map. Because convolution core focuses on expanding channel number and pooling focus on reducing width and height, the model architecture is deeper and wider, while the increase of computation amount slows down.

- Full connection convolution. In the network test phase, three full connections in the training phase are replaced by three convolutions, and the parameters of training are reused in the test, so that the full convolution network can receive any width or height input because there is no full connection limit.

\subsection{Generator model}

The function of the generator is to generate a clear image from the input haze image. It should not only preserve the structure and details of the input image, but also remove the haze as much as possible. The generator introduces the skip connection of symmetry layer in "RESNET" and "u-net" models to break through the bottleneck of information redundancy in the process of dehazing. In addition, the generator discards the method of simply connecting all channels of symmetry layer, and uses the summary method to capture more useful information. The generation process is mainly based on the down sampling operation.

In order to better guide the generator to generate haze-free image, we add a guide module in the generator. The guide maps the high-level features of the data distribution to the low-level representations of the data, and provides the feature mapping to filter the data and parameters by processing the image training set. The process of guide module mainly uses up sampling operation and nonlinear space transfer, absorbs the low-level representation of the training data set, and then outputs the high-level representation of the same data. Specifically, the generator modifies the loss function, and selects the weight for training, so as to obtain a clear image. Fig. 1 shows the generator structure. 


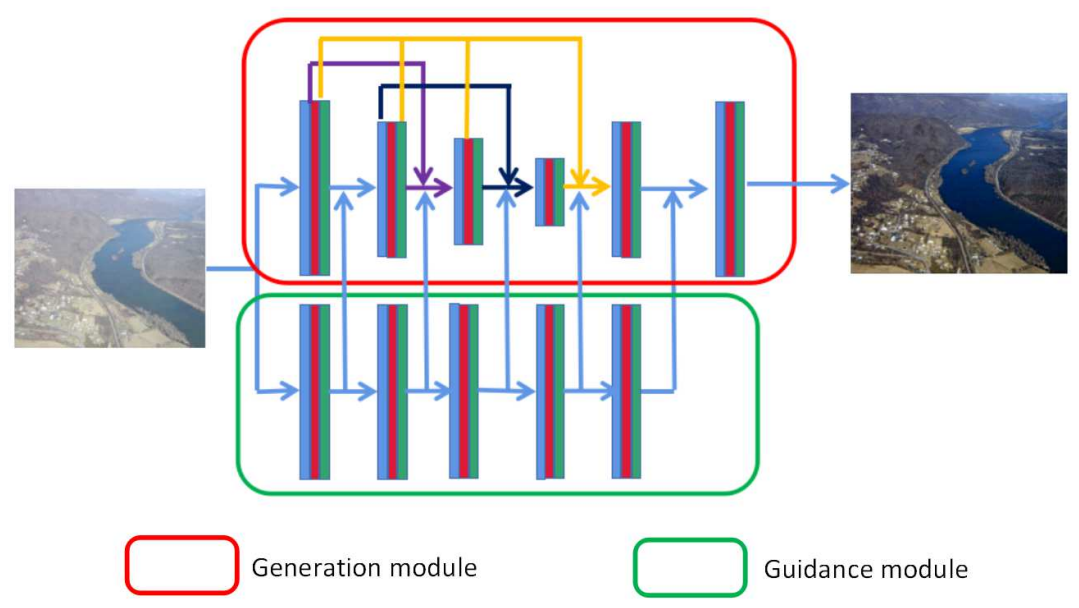

Fig. 1 The structure of generator model.

The main function of generation module is to generate haze-free image. As depicted in Fig. 1, the generation module includes five convolutional layers. These convolutional layers form multi-scale features by fusing varied size filters. They are defined from left to right as follows:

- Conv1: In-channels are three. Out-channels are three. Kernel-size is $1 \times 1$.

- Conv2: In-channels are three. Out-channels are three. Kernel-size is $3 \times 3$.

- Conv3: In-channels are six. Out-channels are three. Kernel-size is $5 \times 5$.

- Conv4: In-channels are six. Out-channels are three. Kernel-size is $7 \times 7$.

- Conv5: In-channels are twelve. Out-channels are three. Kernel-size is $3 \times$ 3.

- Conv6: In-channels are three. Out-channels are three. Kernel-size is $1 \times 1$.

Guidance module provides guidance to the generation module. As depicted in Fig. 1, the compensation module includes five convolutional layers. These layers have the same structure:

- Conv1: In-channels are three. Out-channels are three. Kernel-size is $1 \times 1$.

Every layer of guidance module will output a guide data. These guide data ensure that the generating module will not have large deviation when generating haze-free image.

\subsection{Discriminator model}

The function of discriminant network is to distinguish the real image from the generated image. Like most Gan network structures, the structure design of discriminant network is generally not too complicated. The discriminant 


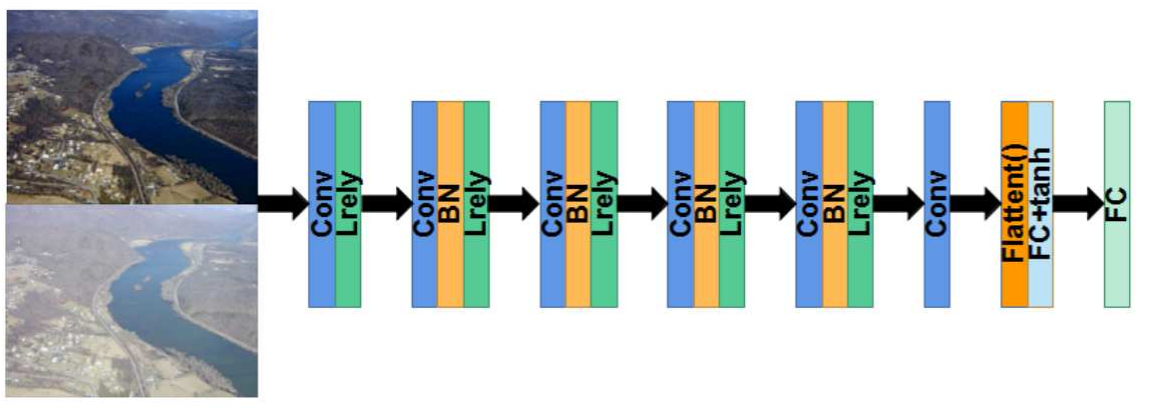

Fig. 2 The structure of discriminator model.

network in this paper is shown in Fig. 2. Different from the original Gan discriminator, as a true false two classifier, the purpose of $D(x)$ of the discriminator in GGan is to approximate the Wasserstein distance between the real sample and the generated sample, which belongs to the regression task. So the network output does not use sigmoid activation function, but directly outputs the result.

The discriminant network includes six convolutional layers. They are defined from left to right as follows:

- Conv1: In-channels are 64. Out-channels are 64. Kernel-size is $4 \times 4$.

- Conv2: In-channels are 64. Out-channels are 128. Kernel-size is $4 \times 4$.

- Conv3: In-channels are 128. Out-channels are 256. Kernel-size is $4 \times 4$.

- Conv4: In-channels are 256. Out-channels are 512. Kernel-size is $4 \times 4$.

- Conv5: In-channels are 512. Out-channels are 1024. Kernel-size is $4 \times 4$.

- Conv6: In-channels are 1024. Out-channels are 1024. Kernel-size is $4 \times 4$.

\subsection{Loss function}

In order to train the proposed network, a loss function is needed to measure the effect of image dehazing. In this paper, a multi task loss function is used, which includes three parts: discrimination loss, image mean square error loss and perception loss Let $\left\{I_{i}, i=1,2, \ldots, N\right\}$ and $\left\{J_{i}, i=1,2, \ldots, N\right\}$ denote the haze image and the corresponding clear image respectively. The GGan training method is to directly use the Adversarial loss function is expressed as:

$$
L_{A}=\frac{1}{N} \sum_{i=1}^{N} \log \left[1-D\left(I_{i}, \tilde{J}_{i}\right)\right]
$$

Where $D$ is the discriminant network, is the output of generator $G$. Discriminators are input in Minibatch mode. In image processing, the whole large training set is divided into several small training sets to improve the computational efficiency and help to train the model quickly. The size of each subset 
of Minibatch's gradient descent is just in the middle of the two extreme cases, and it can be vectorized without waiting for the whole training set to complete.

Only formula 3 cannot restore a clear image, so the perceptual loss based on pre-trained VGG features is introduced to constrain the generator, and the perceptual loss function of VGG features is defined as:

$$
L_{P}=\frac{1}{N} \sum_{i=1}^{N}\left\|F_{i}\left[G\left(I_{i}\right)\right]-F_{i}\left[G\left(J_{i}\right)\right]\right\|_{2}^{2}
$$

Where $G$ is the generating network. $F_{i}$ is the characteristic map of layer $i$. VGG network is pre-trained on ImageNet. Equation 4 can help detail recovery and haze removal, but it introduces artifacts into the restored image, which reduces the quality of the restored image. In order to eliminate artifacts and preserve details and structure, L1 regularization gradient is introduced before the output of the generator and the loss of content-based pixel mode. The define is as follow:

$$
\left.L_{T}=\frac{1}{N} \sum_{i=1}^{N}\left(\| G\left(I_{i}\right)-J_{i}\right)\left\|_{l}+\lambda\right\| \nabla G\left(I_{i}\right) \|_{l}\right)
$$

Where $\left\|\nabla G\left(I_{i}\right)\right\|_{l}$ is the total variation regularization. $\left.\| G\left(I_{i}\right)-J_{i}\right) \|_{l}$ represents content-based direction loss. $\lambda$ is the regularization weight. The loss function can remove the detail artifacts and preserve the details in the image. Finally, these losses are combined to standardize the proposed GGan. The loss function $H$ is defined as:

$$
\hbar=\alpha L_{A}+\beta L_{P}+\gamma L_{T}
$$

Where $\alpha, \beta, \gamma$ represent positive weight. After the generator is modified according to equation 6 , the discriminator is updated by equation 7 .

$$
\max _{D} \frac{1}{N} \sum_{i=1}^{N} \log \left[1-D\left(I_{i}, \tilde{J}_{i}\right)\right]+\log \left[D\left(I_{i}, J_{i}\right)\right]
$$

\section{Experimental results and analysis}

To evaluate the performance of proposed model, we compare it with several advanced single image dehazing algorithms on synthetic datasets and real scene images. The comparison algorithm selected in this paper mainly includes: DCP [39], DehazeNet [21], MSCNN [22], AODNet [40]. DCP is traditional method based on prior. DehazeNet and MSCNN are deep learning methods based on multi-step learning. And AODNet is an end-to-end image dehazing algorithm. 


\subsection{Synthetic experimental data set}

Since there is almost no real haze image data set in the image dehazing, this paper uses the synthesized data set including indoor and outdoor images to train the network. NYU depth data set [41] including only indoor images and Make3D data set [42] of outdoor images are selected as the training data set. Among them, 3000 synthetic images are randomly selected and 300 test images are extracted. Given the clear image $\mathrm{J}$ and the corresponding ground real-time depth $\mathrm{D}$, the random atmospheric light value $A=[n 1, n 2, n 3]$ is generated, where $n \in(0.8,1.0)$. The random value $\rho \in(0.8,1.6)$ is used for each image. Finally, the haze image $I$ is synthesized according to formula 3 . Before the synthesis, the clear image and the corresponding scene depth map are adjusted to a standard size of $512 \times 512$ pixels. Direct synthetic images usually have obvious artifacts. In order to eliminate these artifacts, the better smooth haze image is generated by the method of guided filtering through the filtering depth.

\subsection{Implementation}

In the network training stage, the convolution kernel of each layer is initialized as Gaussian distribution, the bias is initialized as 0 , and the learning rate is initialized as 0.0001. The parameters of loss function are $\alpha=1, \beta=150, \gamma=$ $150, \lambda=10^{-5}$. The model is trained in small batch, and the batch size is 2 . Keras deep learning architecture is used to train the model, RMSprop algorithm is used to optimize the model parameters, and the training epochs is 100. The experimental GPU is Nvidia GTX1080.

\subsection{Analysis of experimental results on synthetic data sets}

In order to verify the dehazing effect of the proposed algorithm, this paper compares the proposed model with the existing classical algorithms in the synthetic data test set. In the synthetic dataset, the clear image corresponding to the haze image is known, so the performance of the model can be evaluated qualitatively and quantitatively.

Fig. 3 shows the dehazing results of a typical two samples in the test set. In the first image, the background is bright, and the difficulty is to remove the haze while preserving the details of the vision. It is not difficult to find that DCP algorithm over dehazing, resulting in color distortion. However, the algorithm based on learning still has the problems of insufficient dehazing degree and loss of image details. The background of the second image is dim, so the restoration of image details is undoubtedly a challenge. As can be seen from the figure, compared with the learning based method, the traditional DCP dehazing result has excessive dehazing. And the learning based methods dehazenet, mscnn, AODNet, compared with proposed method, there is not 


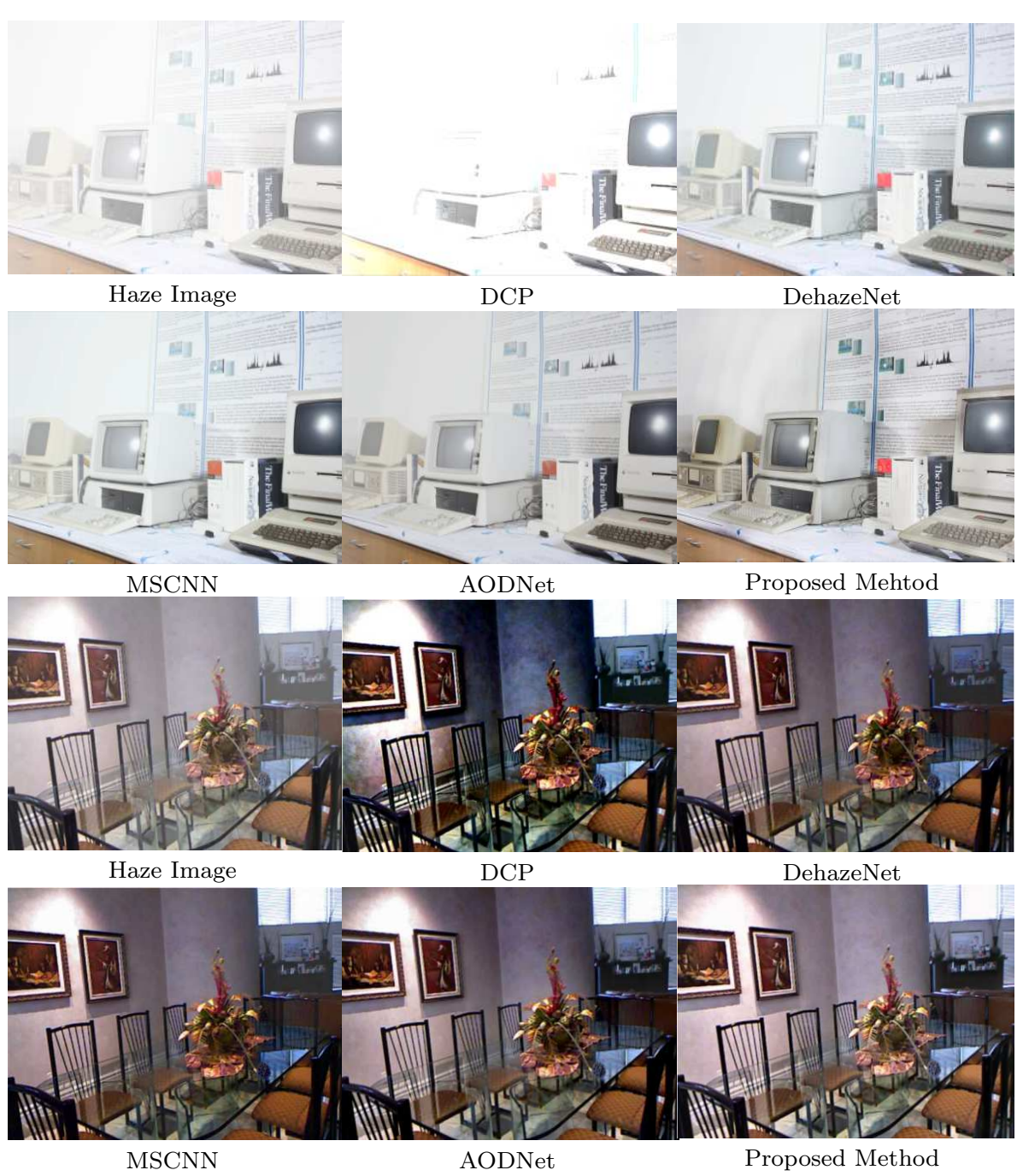

Fig. 3 Qualitative comparisons with state-of-the-art dehazing methods for hazy images on synthetic data sets.

enough dehazing. It can be seen from the results that the proposed algorithm in this paper has strong applicability and better dehazing results.

In order to evaluate the algorithm objectively, this paper uses Peak Signal to Noise Ratio (PSNR) [43] and Structural Similarity index (SSIM) [44] as objective evaluation indexes. PSNR reflects the integrity of image structure information. The higher the PSNR is, the less the image is affected by noise. SSIM reflects the similarity of image structure information. The larger the value is, the closer the image is, and the smaller the distortion difference is. The SSIM and PSNR index results of the five algorithms after dehazing are shown in Table 1. Compared with other algorithms, the SSIM of proposed 
Table 1 Quantitative comparison on the synthetic data-set.

\begin{tabular}{llllll}
\hline & DCP & DehazeNet & MSCNN & AODNet & Proposed \\
\hline PSNR & 18.46 & 21.36 & 20.31 & 19.89 & $\mathbf{2 5 . 8 9}$ \\
SSIM & 0.8808 & 0.8902 & 0.8868 & 0.9000 & $\mathbf{0 . 9 3 8 2}$ \\
\hline
\end{tabular}

algorithm is higher, which shows that the algorithm has better restoration effect in the image edge details. And this algorithm has obvious advantages in PSNR index, which shows that the dehazing image of this algorithm is less affected by noise, the image is clearer, and the dehazing quality is significantly improved.

\subsection{Analysis of experimental results on real images}

The proposed model is trained on the synthetic haze image data set, and the haze removal effect is ideal in the synthetic test set. Because there is still a gap between the real scene and the synthetic haze image in visual perception, in order to verify the generalization ability of proposed method, the proposed method is compared with DCP, DehazeNet, MSCNN and AODNet in the natural scene images. We selected five difficult dehazing images. And the dehazing results are shown in the figure below.

These five haze images are challenging. They either have complex objects or lots of sky and white areas. Surprisingly, the proposed approach has been successful in these challenges. Although DCP method can eliminate some haze, it will produce artifacts and color distortion such as the second image. Due to the inaccuracy of transmittance estimation, DehazeNet and MSCNN still contain more artifacts and haze residues such as the third image and the forth image. AODNet method can generate a clear image as shown in the fifth image, but compared with the dehazing image generated by proposed algorithm, there are more details of unclear objects, the color of dehazing image is dark, and the sky part of the image has slight color distortion. Compared with other dehazing methods (DCP, DehazeNet, MSCNN and AODNet ), the advantages of proposed method are that the detail information of dehazing image is preserved completely, the color recovery is more natural, and the degree of dehazing is moderate. Therefore, the algorithm in this paper is better than other contrast algorithms in natural haze image processing, and has strong applicability.

\section{Conclusions}

This paper presents a Guided Generative Adversarial Dehazing Network(GGADN). In GGADN the generator and discriminator architecture are modified. The synthetic data set is trained by end-to-end training neural network. The loss function is modified by using the pre-trained VGG feature and L1-regularization gradient. Sigmoid function is introduced to the last layer of discriminator for 


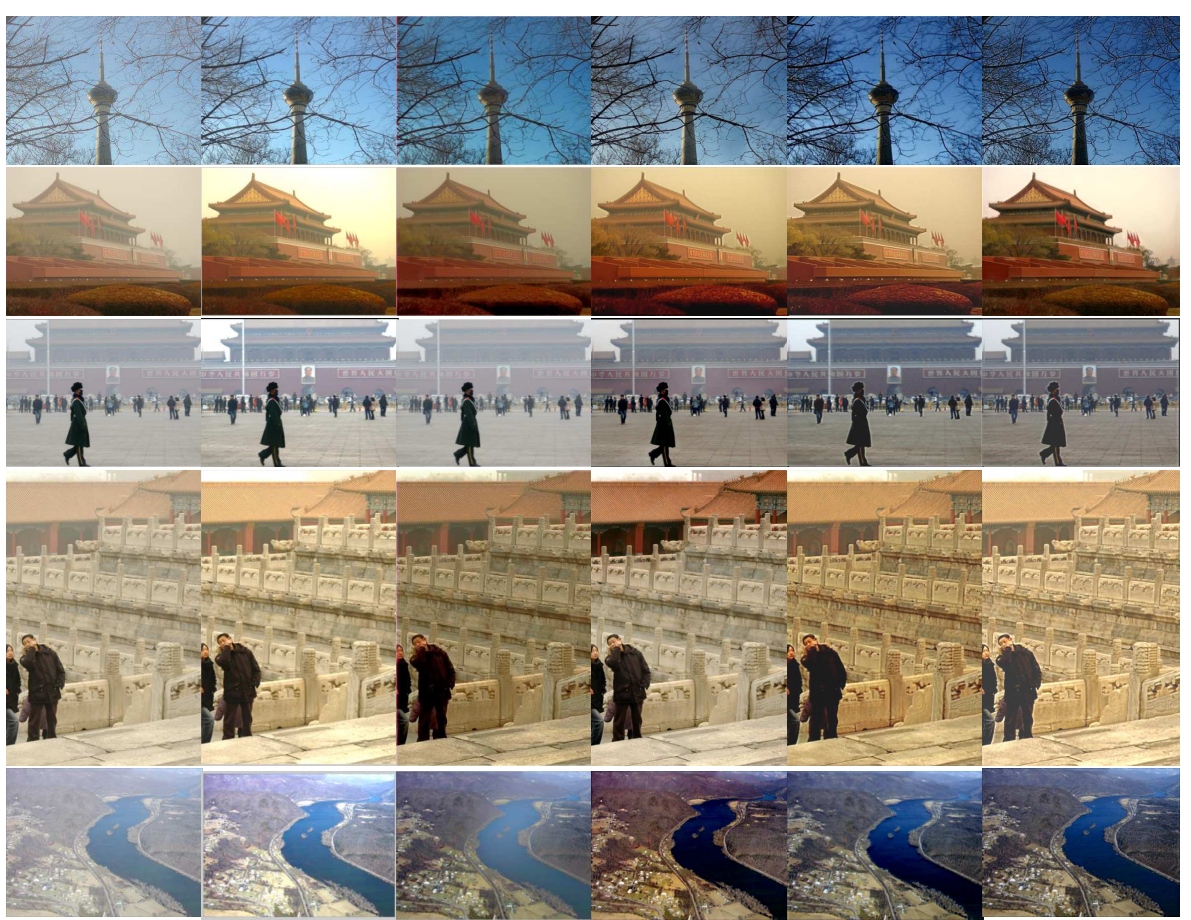

Haze Image

DCP

DehazeNet

MSCNN

AODNet

Proposed

Fig. 4 Qualitative comparisons with state-of-the-art dehazing methods for hazy images on real data sets.

feature mapping. From the dehazing results of synthetic images and real images, proposed method is better than the state-of-the-art dehazing methods. And the image after dehazing is clearer in detail.

In the future, we will explore in at least two directions based on proposed method. The first one is to explore the clear image without reference for training the dehazing network $[45,46,51,52]$. The second is to explore video dehazing [47-50].

\section{Acknowledgments}

This work is supported by Science and Technology Department of Hubei Province, China (Grant No. 2014CFB383).

\section{Compliance with Ethical Standards}

I. Ethical approval

- This manuscript was not submitted to more than one journal for simultaneous consideration. 
- The submitted work is original and has not been published elsewhere in any form or language, unless the new work concerns an expansion of previous work.

- This study is not split up into several parts to increase the quantity of submissions and submitted to various journals or to one journal over time.

- Concurrent or secondary publication is sometimes justifiable, provided certain conditions are met.

- Results are presented clearly, honestly, and without fabrication, falsification or inappropriate data manipulation (including image based manipulation). Authors adhere to discipline-specific rules for acquiring, selecting and processing data.

- No data, text, or theories by others are presented as if they were the author's own.

II. Funding details

This work is supported by Science and Technology Department of Hubei Province, China (Grant No. 2014CFB383).

\section{Conflict of interest}

The authors declare that they have no conflicts of interest.

\section{Informed Consent}

Informed consent was obtained from all individual participants included in the study.

\section{Authorship contributions}

All authors contributed to the study conception and design. Material preparation, data collection and analysis were performed by Jiang Zhang and Wanjuan Song. The first draft of the manuscript was written by Jian Zhang and all authors commented on previous versions of the manuscript. All authors read and approved the final manuscript.

\section{References}

1. Voulodimos, Athanasios, et al. "Deep learning for computer vision: A brief review." Computational intelligence and neuroscience (2018).

2. Khan, Salman, et al. "A guide to convolutional neural networks for computer vision." Synthesis Lectures on Computer Vision 8.1: 1-207 (2018). 
3. Janai, Joel, et al. "Computer vision for autonomous vehicles: Problems, datasets and state of the art." Foundations and Trends( $\mathrm{R}$ in Computer Graphics and Vision 12.1-3: 1-308 (2020)

4. O'Mahony, Niall, et al. "Deep learning vs. traditional computer vision." Science and Information Conference. Springer, Cham (2019).

5. Nanni, Loris, Stefano Ghidoni, and Sheryl Brahnam. "Handcrafted vs. non-handcrafted features for computer vision classification." Pattern Recognition 71: 158-172 (2017).

6. Kendall, Alex, and Yarin Gal. "What uncertainties do we need in bayesian deep learning for computer vision?." arXiv preprint arXiv:1703.04977 (2017).

7. Zhang, Jian, Fazhi He, and Yilin Chen. "A new haze removal approach for sky/river alike scenes based on external and internal clues." Multimedia Tools and Applications 79.3: 2085-2107 (2020).

8. Dudhane, Akshay, and Subrahmanyam Murala. "RYF-Net: Deep fusion network for single image haze removal." IEEE Transactions on Image Processing 29 (2019): 628-640.

9. Zhang, Jing, et al. "Fast haze removal for nighttime image using maximum reflectance prior." Proceedings of the IEEE conference on computer vision and pattern recognition. (2017).

10. Chen, Wei-Ting, et al. "PMHLD: patch map-based hybrid learning DehazeNet for single image haze removal." IEEE Transactions on Image Processing 29: 6773-6788 (2020).

11. Yeh, Chia-Hung, Chih-Hsiang Huang, and Li-Wei Kang. "Multi-scale deep residual learning-based single image haze removal via image decomposition." IEEE Transactions on Image Processing 29: 3153-3167 (2019).

12. Yang, Xi, et al. "Single image haze removal via region detection network." IEEE Transactions on Multimedia 21.10: 2545-2560 (2019).

13. Shen, Huanfeng, et al. "A Spatial-Spectral Adaptive Haze Removal Method for Visible Remote Sensing Images." IEEE Transactions on Geoscience and Remote Sensing 58.9: 6168-6180 (2020).

14. Zhao, Shiyu, et al. "RefineDNet: A Weakly Supervised Refinement Framework for Single Image Dehazing." IEEE Transactions on Image Processing 30: 3391-3404 (2021).

15. Li, Boyun, et al. "You only look yourself: Unsupervised and untrained single image dehazing neural network." International Journal of Computer Vision : 1-14 (2021).

16. Shyam, Pranjay, Kuk-Jin Yoon, and Kyung-Soo Kim. "Towards Domain Invariant Single Image Dehazing." arXiv preprint arXiv:2101.10449 (2021).

17. Hou G, Li J, Wang G, et al. A novel dark channel prior guided variational framework for underwater image restoration $[\mathrm{J}]$. Journal of Visual Communication and Image Representation, 66: 102732 (2020).

18. Dharejo, Fayaz Ali, et al. "A remote-sensing image enhancement algorithm based on patch-wise dark channel prior and histogram equalisation with colour correction." IET Image Processing 15.1: 47-56 (2021).

19. Iwamoto, Yutaro, Naoaki Hashimoto, and Yen-Wei Chen. "Real-Time Haze Removal Using Normalised Pixel-Wise Dark-Channel Prior and Robust Atmospheric-Light Estimation." Applied Sciences 10.3: 1165 (2020)

20. Zhang, Jian, Feihu Feng, and Wanjuan Song. "A compensation textures dehazing method for water alike area." The Journal of Supercomputing 77.4: 3555-3570 (2021).

21. Cai, Bolun, et al. "Dehazenet: An end-to-end system for single image haze removal." IEEE Transactions on Image Processing 25.11: 5187-5198 (2016).

22. Ren, Wenqi, et al. "Single image dehazing via multi-scale convolutional neural networks." European conference on computer vision. Springer, Cham: 154-169 (2016).

23. Li, Boyi, et al. "Aod-net: All-in-one dehazing network." Proceedings of the IEEE international conference on computer vision. 2017:4770-4778 (2017).

24. McCartney, Earl J. "Optics of the atmosphere: scattering by molecules and particles." New York (1976).

25. Ju, Mingye, et al. "IDE: Image Dehazing and Exposure Using an Enhanced Atmospheric Scattering Model." IEEE Transactions on Image Processing 30: 2180-2192 (2021).

26. Ju, Mingye, Dengyin Zhang, and Xuemei Wang. "Single image dehazing via an improved atmospheric scattering model." The Visual Computer 33.12: 1613-1625 (2017).

27. Dai, Chenggang, et al. "Single hazy image restoration using robust atmospheric scattering model." Signal Processing 166: 107257 (2020). 
28. Goodfellow, Ian J., et al. "Generative adversarial networks." arXiv preprint arXiv:1406.2661 (2014).

29. Karras, Tero, et al. "Training generative adversarial networks with limited data." arXiv preprint arXiv:2006.06676 (2020).

30. Schonfeld, Edgar, Bernt Schiele, and Anna Khoreva. "A u-net based discriminator for generative adversarial networks." Proceedings of the IEEE/CVF Conference on Computer Vision and Pattern Recognition. 2020.

31. Gui, Jie, et al. "A review on generative adversarial networks: Algorithms, theory, and applications." arXiv preprint arXiv:2001.06937 (2020).

32. Yamamoto, Ryuichi, Eunwoo Song, and Jae-Min Kim. "Parallel WaveGAN: A fast waveform generation model based on generative adversarial networks with multi-resolution spectrogram." ICASSP 2020-2020 IEEE International Conference on Acoustics, Speech and Signal Processing (ICASSP). IEEE (2020).

33. Zhang, Wei, et al. "Machinery fault diagnosis with imbalanced data using deep generative adversarial networks." Measurement 152 : 107377 (2020).

34. Lin, Jiacheng, Yang Li, and Guanci Yang. "FPGAN: Face de-identification method with generative adversarial networks for social robots." Neural Networks 133: 132-147 (2021).

35. Deldjoo, Yashar, Tommaso Di Noia, and Felice Antonio Merra. "A survey on adversarial recommender systems: from attack/defense strategies to generative adversarial networks." ACM Computing Surveys (CSUR) 54.2: 1-38 (2021).

36. Sohn, Kihyuk, Honglak Lee, and Xinchen Yan. "Learning structured output representation using deep conditional generative models." Advances in neural information processing systems 28: 3483-3491 (2015).

37. Engin, Deniz, Anil Genç, and Hazim Kemal Ekenel. "Cycle-dehaze: Enhanced cyclegan for single image dehazing." Proceedings of the IEEE Conference on Computer Vision and Pattern Recognition Workshops (2018).

38. Du, Yixin, and Xin Li. "Recursive deep residual learning for single image dehazing." Proceedings of the IEEE Conference on Computer Vision and Pattern Recognition Workshop (2018) .

39. He, Kaiming, Jian Sun, and Xiaoou Tang. "Single image haze removal using dark channel prior." IEEE transactions on pattern analysis and machine intelligence 33.12: 2341$2353(2010)$.

40. Qian, Wen, Chao Zhou, and Dengyin Zhang. "FAOD-Net: a fast AOD-Net for dehazing single image." Mathematical Problems in Engineering 2020 (2020).

41. Huynh, Lam, et al. "Guiding monocular depth estimation using depth-attention volume." European Conference on Computer Vision. Springer, Cham (2020).

42. Sagar, Abhinav. "Monocular Depth Estimation Using Multi Scale Neural Network And Feature Fusion." arXiv preprint arXiv:2009.09934 (2020).

43. Helmrich, Christian R., et al. "Xpsnr: A Low-Complexity Extension of The Perceptually Weighted Peak Signal-To-Noise Ratio For High-Resolution Video Quality Assessment." ICASSP 2020-2020 IEEE International Conference on Acoustics, Speech and Signal Processing (ICASSP). IEEE (2020).

44. Jin, Chanwoo, et al. "Similarity measurement on human mobility data with spatially weighted structural similarity index (SpSSIM)." Transactions in GIS 24.1: 104-122 (2020).

45. Huang, Xin, et al. "A two-sided matching model for complex equipment production of military-civilian merging platform with reference effects." Soft Computing: 1-23 (2021).

46. Wang, Hui, et al. "A novel multi-objective optimization algorithm for the integrated scheduling of flexible job shops considering preventive maintenance activities and transportation processes." Soft Computing 25.4: 2863-2889 (2021).

47. Sekh, Arif Ahmed, et al. "Video trajectory analysis using unsupervised clustering and multi-criteria ranking." Soft Computing 24: 16643-16654 (2020).

48. Rani, Shalli, et al. "Detection of shilling attack in recommender system for YouTube video statistics using machine learning techniques." Soft Computing: 1-13 (2021).

49. Preethaa, KR Sri, and A. Sabari. "Intelligent video analysis for enhanced pedestrian detection by hybrid metaheuristic approach." Soft Computing: 1-9 (2020).

50. Ahmad, M. Z., and J. F. Peters. "Fuzzy hyperconnected proximity spaces and fuzzy summability over CW complexes. Application of Smirnov fuzzy similarity in video analysis." Soft Computing: 1-12 (2020). 
51. Caviglione, Luca, et al. "Deep reinforcement learning for multi-objective placement of virtual machines in cloud datacenters." Soft Computing: 1-20 (2020).

52. An, Fengping. "Image classification algorithm based on stacked sparse coding deep learning model-optimized kernel function nonnegative sparse representation." Soft Computing 24.22: $16967-16981$ (2020) 
Figures

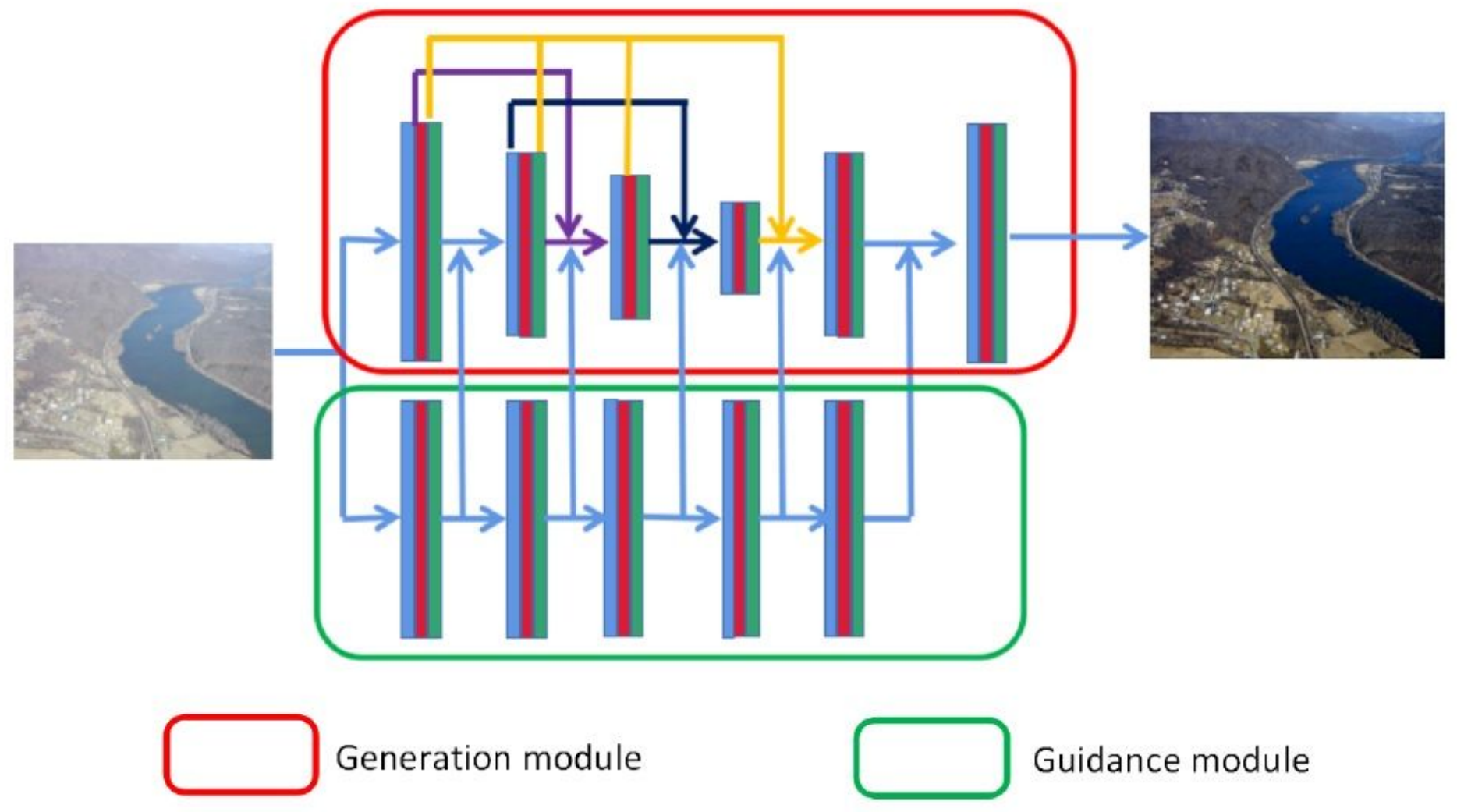

Figure 1

The structure of generator model.

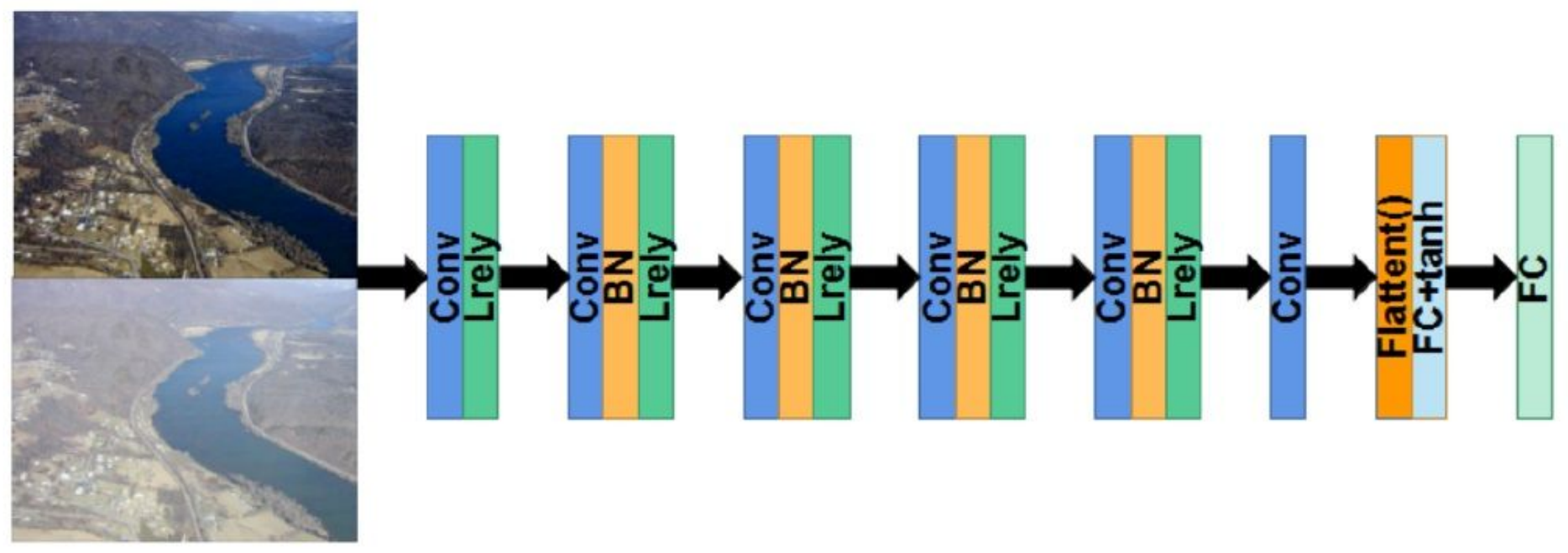

Figure 2

The structure of discriminator model. 


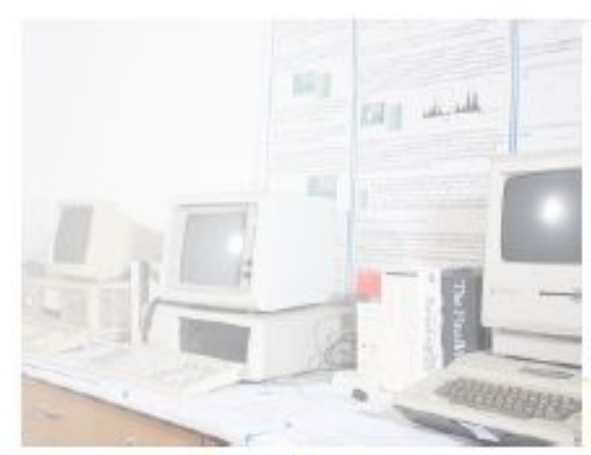

Haze Image

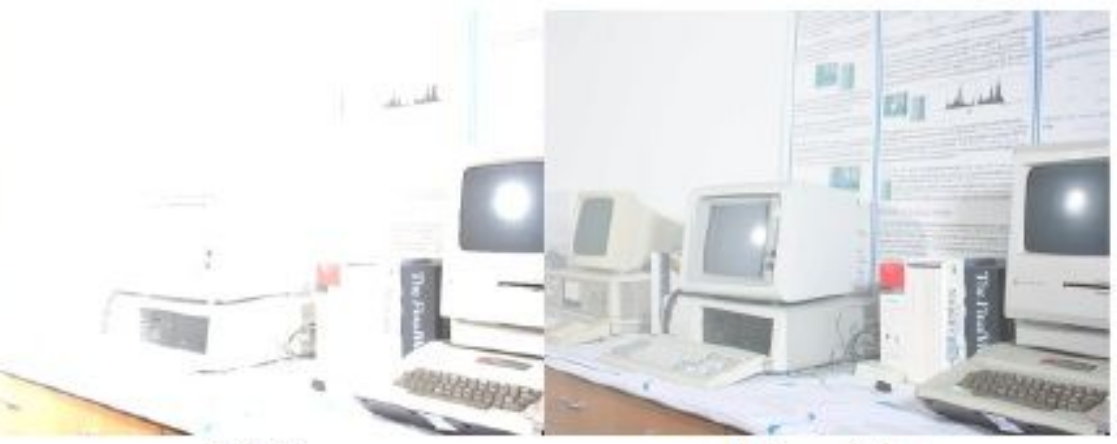

DCP

DehazeNet

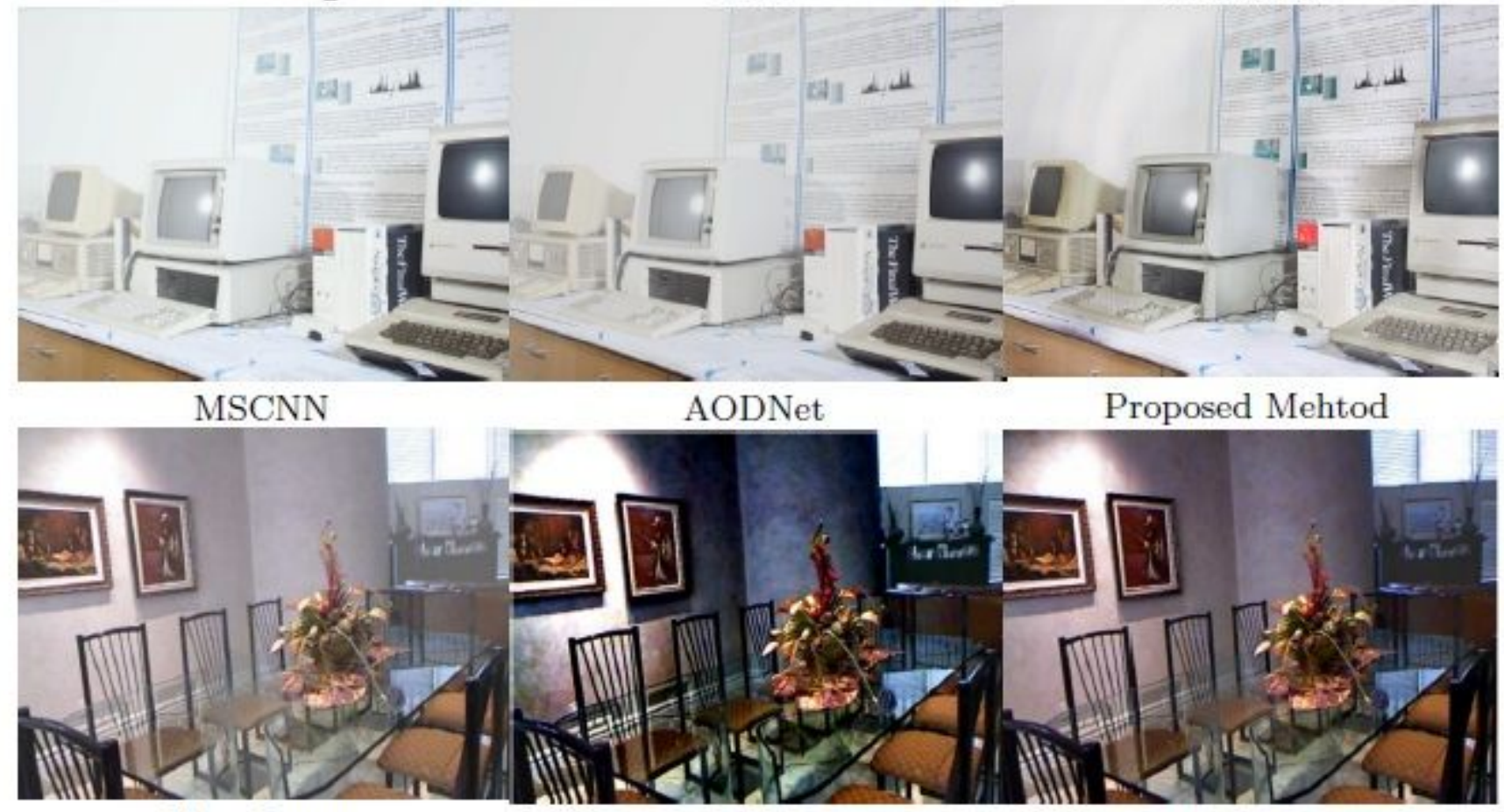

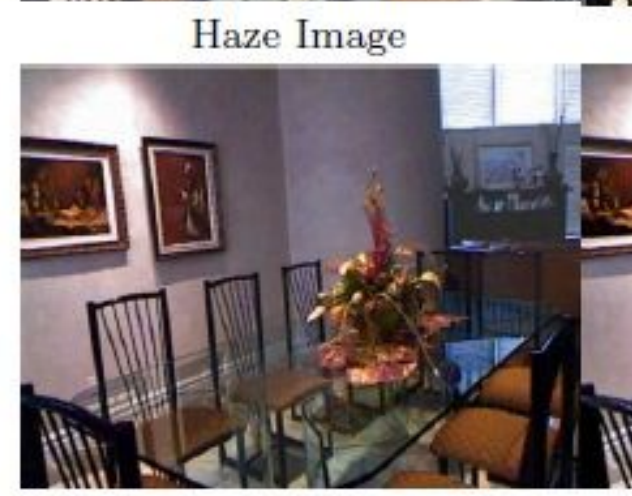

MSCNN
DCP

DehazeNet

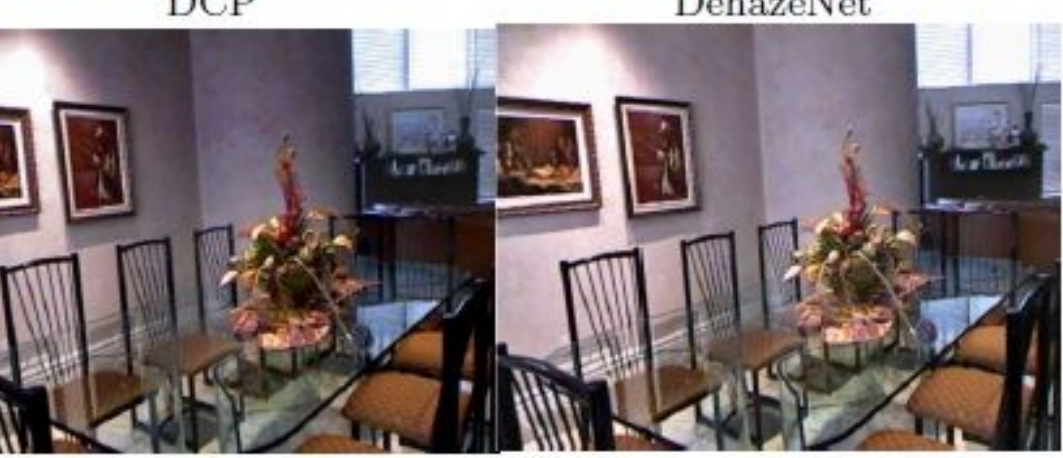

AODNet

Figure 3

Qualitative comparisons with state-of-the-art dehazing methods for hazy images on synthetic data sets. 


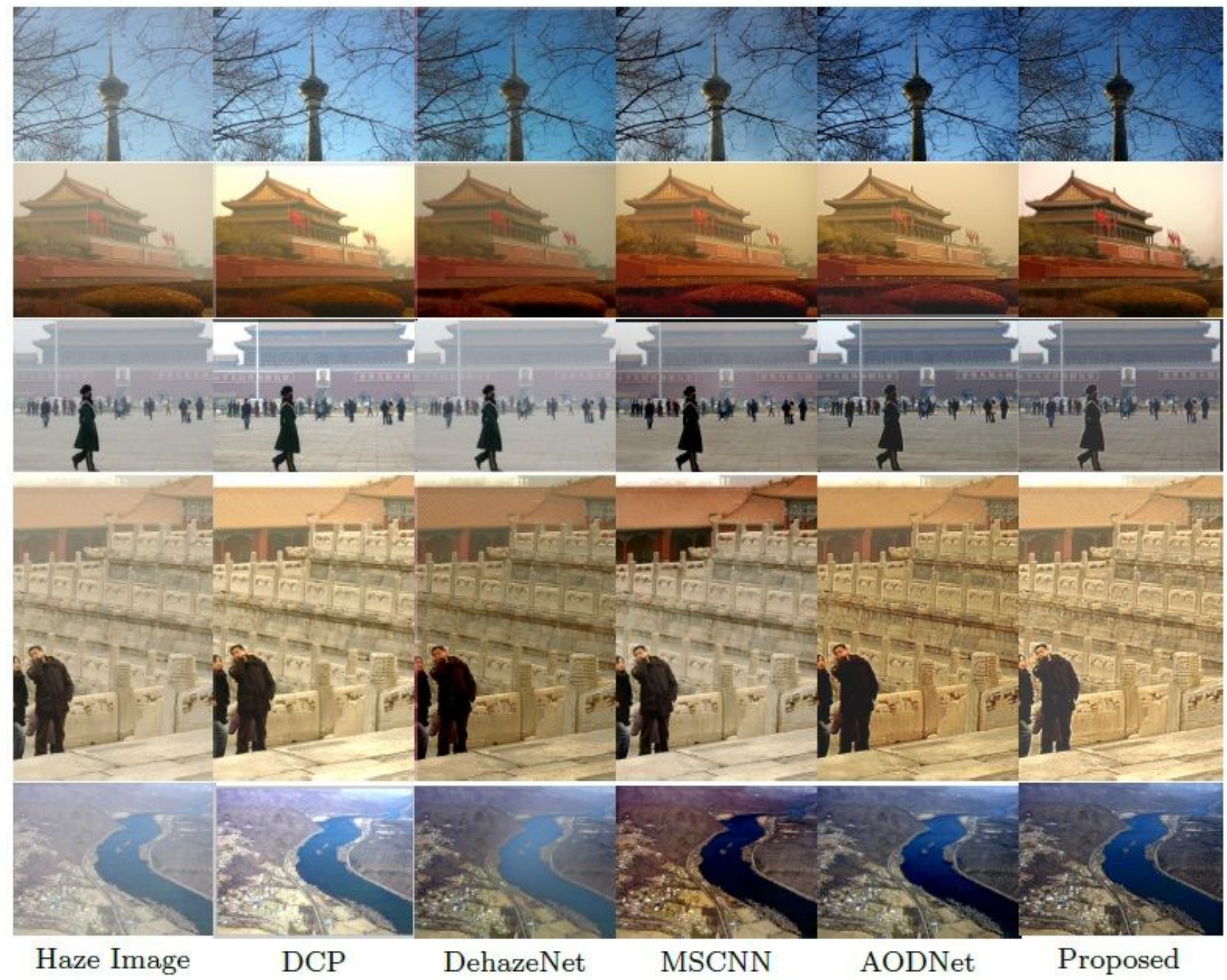

Figure 4

Qualitative comparisons with state-of-the-art dehazing methods for hazy images on real data sets. 